\title{
LA CONTRIBUCIÓN DE PHILIPP \\ MELANCHTHON, PRAECEPTOR \\ GERMANIAE, A LA LENGUA \\ Y A LA ENSEÑANZA. ENTRE LA CULTURA \\ HUMANISTA EUROPEA Y LA REFORMA \\ ALEMANA
}

\section{The contribution of Philipp Melanchthon, Praeceptor Germaniae, to language and education. Between European bumanist culture and German reform}

Juan Luis Monreal Pérez

Departamento de Traducción e Interpretación. Universidad de Murcia

Correo-e: jlmonreal@um.es

Recepción: 26 de noviembre de 20I5. Envío a informantes: 5 de enero de 2016.

Aceptación definitiva: 17 de junio de 2016

Resumen: Aunque pueda calificarse a Melanchthon de hombre eminente, sin embargo, no es suficientemente conocido, ni traducido, ni estudiado fuera de Alemania. Su pensamiento acerca de la lengua y de la enseñanza no se redujo a mostrar su importancia. Fue más lejos que esto, ya que le preocupó mucho el uso didáctico de las mismas. Su preocupación fundamental por mejorar el aprendizaje de las lenguas se focalizó en las lenguas clásicas, especialmente en el latín, sin que esto supusiera darle la espalda a las lenguas vernáculas, en este caso al alemán. El hecho de que para Melanchthon las Letras y los grandes escritores de la Antigüedad fueran su preocupación principal, explica el reconocimiento público que Alemania le hizo, nombrándole Praeceptor Germaniae.

PAlabras Clave: lengua; enseñanza; cultura humanista europea; reforma protestante; Ratio Studiorum de la reforma protestante. 
AвSTRACт: Although Melanchthon could be described as an eminent man, nevertheless, he is neither sufficiently known, translated, nor studied outside Germany. Melanchthon's thoughts about language and education were not limited to showing their importance. He went further than this, because he worried greatly about the didactic use of both. His fundamental concern to improve language learning focused on classical languages, especially Latin, without this supposing turning his back on vernacular languages, German in this case. The fact that for Melanchthon bumanities and the great writers of antiquity were his main concern, explain the public recognition Germany gave in naming him Praeceptor Germaniae.

KEY WORDS: language; education; European humanist culture; Protestant Reformation; Ratio Studiorum of the Protestant Reformation.

\title{
I. Introducción
}

\begin{abstract}
Philip Melanchthon le tocó asumir un papel secundario a lo largo de A su vida, razón por la que no es suficientemente conocido, ni traducido, 1 ni estudiado fuera de Alemania, independientemente de que pueda calificársele como hombre eminente del Humanismo renacentista y como figura luminosa que encarna el ideal de aquello que llamamos humanismo cristiano o, como decía de él Lutero, que no solo estaba llamado a la inmortalidad, sino que también merecía su inclusión en el canon de la Iglesia (Stroh, 20I2: 224 y 234). Aparte de que su personalidad y su educación le condicionaron a ocupar una posición discreta, fue sobre todo la figura de Lutero, bajo cuya sombra vivió la mayor parte de su vida, la que hizo que Melanchthon estuviera de forma natural en un segundo plano el largo tiempo que vivieron y trabajaron juntos, en la condición de colaborador cercano y fiel a Lutero.

El presente artículo, ubicado en el contexto en el que se desarrolla la vida, obra y trabajo de Melanchthon, quiere hacer una modesta contribución de su aportación a la lengua y a la enseñanza, cuestiones centrales para el Humanismo renacentista en general y, cómo no, para la cultura humanista de la Reforma, razón por la que conviene recordar, con carácter previo, las ansias de reforma que el Humanismo renacentista conllevó y que se materializaron, entre otros ámbitos, en el cultivo y amor por la lengua y la enseñanza.
\end{abstract}

\section{Philipp Melanchthon, hombre humanista}

En Melanchthon, su condición de hombre reformador está relacionada con su condición de hombre humanista; aspectos estrechamente relacionados en su trayectoria de vida. Desde el inicio de su formación académica hasta su total compromiso con la Reforma protestante, en todo lo que escribe y hace, el espíritu humanista es una constante en su vida, al manifestarse de manera firme en su

Sтroh, W.: El latín ha muerto. ;Viva el latín!, Barcelona, Ediciones del Subsuelo, 2012. 
acción la unión de lengua, razón y moralidad (Stroh, 20I2: 237)². Esto explica en Melanchthon su permanente compromiso humanista dentro del llamado Humanismo renacentista (Pérez, I99I: 1333; Burckhardt, 1984: 1694 y Rico, 1993: 19), que se manifiesta tanto en su proceso formativo como en la elaboración de su pensamiento y en la intensa actividad que desarrolla.

Melanchthon obtuvo el título de bachiller en Artes clásicas en la Universidad de Heidelberg (I5II), y más tarde (I5I4), en la Universidad de Tubinga, recibió el título de Maestro de Artes, cursando Filosofía. Fue en esta universidad donde realmente se sensibilizó y tomó partido por el Humanismo, influyendo en ello el círculo de amigos que frecuentó y que la mayoría de ellos cursaban estudios de humanismo; de entre ellos conviene reseñar a Johannes Öcolampadius, quien más tarde sería un importante reformador suizo, y Ambrosius Blarer, quien ocuparía también una posición relevante en la corriente reformadora de Wittenberg. Con ellos Melanchthon abundaría en el conocimiento de los autores griegos clásicos, seguiría profundizando en la lógica escolástica, a través de la Dialéctica de Rudolf Agrícola, publicada en I515, y estudiaría las aportaciones que nuevas ciencias, como la astronomía y astrología, hacían al desarrollo del saber.

Fruto de su formación y de sus contactos en Tubinga, los escritos tempranos de Melanchthon se ven ya marcados por el espíritu humanista, tal como señala Horst F. Rupp, reconocido especialista del reformador germano:

En este clima intelectual tan estimulante, no tardaron en aparecer nuevas obras suyas: así, Reuchlin ${ }^{6}$ le encargó a Melanchthon y a un compañero que escribieran el prefacio de Clarorum virorum epistolae [Epístolas de varones ilustres] (I5I4), en el cual el humanista se defendió de los ataques de los llamados «Obscurantistas»; a esto le siguió una edición de las obras de Terencio (I516) con una introducción a la historia de la comedia, la obra filológica más importante de Melanchthon. Hacia el final de 1517, con motivo de una celebración académica en Tubinga, pronunció un discurso sobre el tema de las disciplinas que se enseñaban en la universidad, en el que proponía añadir la historia y la poesía a las originales siete artes liberales (De septem artibus liberalibus), el trivium (gramática, retórica y dialéctica) y el quadrivium (aritmética, geometría, música y astronomía), cuyo estudio en la Facultad de Artes supuestamente preparaba a los estudiantes para las tres facultades superiores de medicina, derecho y teología (Rupp, 1996: 662).

Op. cit.

3 PÉrez, J.: «El hombre del Renacimiento», en vv. AA.: El siglo de Fray Luis de León. Salamanca y el Renacimiento, Salamanca, Universidad de Salamanca, I99I, pp. I3-24.

4 Burckhardt, J.: La cultura del Renacimiento en Italia, México, Editorial Porrúa, 1984.

5 Rico, F.: El sueño del Humanismo (De Erasmo a Petrarca), Madrid, Alianza Universidad, 1993.

6 Jahannes Reuchlin, tío abuelo de Melanchthon y reputado humanista que contribuyó considerablemente al estudio del hebreo bíblico y a la libertad de la investigación científica, influyó bastante en la trayectoria académica de Melanchthon, orientándole en su formación clásica, apoyándole en la consecución de la cátedra de griego de Wittenberg y dándole la forma griega a su apellido, convirtiéndolo en Philipp Melanchthon.

Rupp, H. F.: «Philipp Melanchthon (I497-1560)», Revista Perspectivas, vol. xxvı, n. ${ }^{\circ}$ 3, septiembre (1996), pp. 659-669. 
La etapa de Melanchthon como docente en la Universidad de Tubinga, pese a su juventud, le permitió, por una parte, iniciar la elaboración de su pensamiento humanístico a través de los trabajos mencionados, pero también fue el punto de partida para la producción de materiales para la escuela, respondiendo así a las necesidades didácticas que reclamaba. Resultado de ello es la edición que hace de la obra latina de Terencio adaptada a los niños y las gramáticas de latín y griego, que tendrían un considerable éxito.

La llegada de Melanchthon a Wittenberg para ocupar la cátedra de Griego, que precisamente fue creada por Federico el Sabio, elector de Sajonia, como parte de la reforma humanística de esa universidad, le permitió desarrollar su actividad docente orientándola claramente bajo el espíritu humanista. Un buen dato que confirma tal orientación fue que su primera lección universitaria (28 de agosto de I5I8) tuvo como objeto el tema de la Mejora de los estudios para los jóvenes (De corrigendis adolescentiae studiis), dejando con esto bien claro el espíritu humanista que le guiaba. Un tiempo más tarde, y siguiendo el mismo espíritu humanista, Melanchthon pronunció un memorable discurso titulado El Elogio de la elocuencia (Encomium eloquentiae) en el que, siguiendo la tradición humanista clásica (Ovidio, Cicerón), defiende la importancia de este arte para bien expresarse, para la comprensión de textos, tanto clásicos latinos como bíblicos, para el estudio de lenguas y, en definitiva, para la educación del espíritu. Con esta aportación, Melanchthon profundiza en la corriente humanista, asumiendo la tradición de los Studia Humanitatis, actitud y perspectiva que manifestará a lo largo de su vida y en su notable contribución a la Reforma. Ello explica que el espíritu humanista de Melanchthon sea el resultado de la combinación articulada de lengua, razón y educación moral/intelectual.

Por todo ello, Melanchthon, en lo fundamental, participaba de los principios que caracterizaban al movimiento humanista renacentista que se conforma en la continuidad entre la tradición medieval y el pensamiento humanista (Vilanou, 20I2: I40): retorno a las fuentes clásicas, defensa de las lenguas clásicas, crítica del degenerado uso que se hacía del latín e interés por los saberes que favorecían la modernización de la sociedad, tales como la historia, las ciencias naturales y las matemáticas (Labrador, 1984: 18)9.

La condición de Melanchthon, como hombre reformador, no le supuso renunciar a su espíritu humanista (Pena, 2002:IO) ${ }^{\mathrm{IO}}$. Incluso en los momentos y en las cuestiones religiosas de mayor confrontación, supo situarse en posiciones intermedias o en terceras vías que no contradecían su trayectoria y espíritu humanistas; tal es el caso de la cuestión del libre albedrío o, incluso, de su habitabilidad con Lutero en los momentos de mayor tensión doctrinal.

8 Vilanou, C.: «El humanismo de Eiximenis: saber, ciudad y cortesía», Historia de la Educación, vol. 3I (20I2), pp. 23-32.

9 LABRADOR, C.: «Felipe Melanchthon, iniciador de la segunda enseñanza humanística», $R e-$ vista Miscelánea Comillas, n. ${ }^{\circ} 2$ (1984), pp. 17-36.

1o Pena, P.: Las fuentes de la teología en Felipe Melanchthon. Su doctrina sobre los lugares teológicos comparada con la doctrina católica de M. Cano y la tradición medieval. Extracto de la tesis, Universidad Pontificia de Salamanca, 2002. 


\section{Philipp Melanchthon, hombre reformador en el campo de la enseñanza}

Fue en el ámbito de la política escolar en el que Melanchthon expresó, especialmente, su carácter reformador. Uno de los principales precedentes para que expresara su voluntad de reformar la educación y de darle un giro humanista a los programas educativos políticamente influyentes, tal y como expresó en la inauguración de sus clases en la Universidad de Wittenberg, fue la aparición en I444 de la obra de Lorenzo Valla Elegantiarum Latinae linguae libri sex. Eiusdem de reciprocatione sui, et suus, libellus. Por otro lado, en la segunda mitad del siglo, crecía la crítica a la obra Doctrinale y a su autor Alexander de Villa Dei" en base a objeciones metodológicas y al abuso que de esa gramática se hacía en la enseñanza. Alexander Clemen (1932), según Rainer Kößling (1999), constata dicha crítica en un artículo titulado Una sátira no conocida al Doctrinale de Alexander de Villa Dei $i^{\text {i2 }}$ cuando tacha a dicho manual de no ser efectivo a la hora de facilitar la comprensión de textos escritos en latín, al decir: «Der Gebrauch des Grammatiklehrbuchs im Untericht war weithin zum Selbstzweck geraten und im Hinblick auf das Ziel einer rasch einsetzenden Textlektüre nicht effektiv» ${ }^{13}$

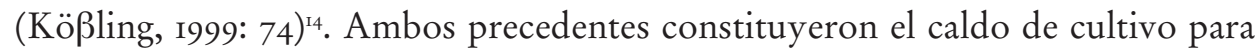
que Melanchthon mostrara su voluntad decidida de reformar la educación.

Resultado de esta preocupación y compromiso de Melanchthon en este campo es la producción de libros de texto que necesitaban las escuelas y las universidades en Alemania en todos los ámbitos del saber, como gramática griega y latina, retórica, dialéctica, autores latinos, ciencias naturales, física, psicología, ética, etc. En general sus obras ${ }^{15}$ y, especialmente, su Gramática latina (Grammatica D. Philippi latina cum exercitio) era de óptima calidad didáctica, ya que desde un principio estaba concebida para su uso académico y para ser utilizada por profesores motivados. De hecho, se tiene constancia de que hasta 1757 dicha gramática se editó más de 200 veces y su uso era habitual en las escuelas, tal como señala el siguiente fragmento: «So weist, um ein Beispiel zu nennen, der älteste erhaltene Stundenplan der Leipziger Nicolaischule aus dem Jahre 1574 für die III Klasse

"Villa, D. de, A.: Das Doctrinale. Edición crítico-exegética adaptada por Dieter Reichlin, Berlin, A. Hofmann \& comp., I893.

${ }_{12}$ Clemen, A.: «Una sátira no conocida al Doctrinale de Alexander De Villa Dei», Revista para la Historia de la Educación y de la Enseñanza, n. ${ }^{\circ}$ 22. Reimpresión CIK Sch 5 (1932), pp. 676-684.

${ }_{13}$ Traducción propia como en el resto de las que figuran a pie de página: «El uso de dicho manual de gramática en clase había llegado a ser un fin en sí mismo y no era efectivo para asegurar la inmediatez de la incipiente actividad de leer un texto».

${ }_{14}$ KöBling, R.: «Philipp Melanchthon als Grammatiker der lateinischen Sprache», en WARTENBerg, G. et al: Werk und Rezeption Philipp Melanchthon in Universität und Schule bis ins I8. Jabrbundert, Universität Leipzig, Evangelische Verlagsanstalt, 1999.

${ }^{15}$ La producción científica de Melanchthon es suficientemente amplia. A las temáticas ya mencionadas, conviene añadir los textos Enquiridion elementorum puerilium y Cathechesis Puerilis, por su carácter didáctico, aparte de las abundantes cartas que intercambió con relevantes personajes de aquella época (reyes, gobernantes y humanistas). Para consultar los textos de Melanchthon a través de fuentes primarias $c f$. Negrín, O.: «Fuentes para el estudio del Humanismo pedagógico en Europa», Historia de la Educación, vol. 3I (2012), pp. 319-338, pp. 327-328. 
"Grammatica D. Philippi latina cum exercitio" aus, montags bis freitags von 8-9 Uhr» ${ }^{16}$ (Kößling, 1999: 8I) $)^{17}$.

Melanchthon incorporó dicha gramática a su política educativa, al elaborarla con perspectiva pedagógica para ser utilizada por docentes y alumnos. En su elaboración contó con la colaboración de expertos en didáctica y en todos los ámbitos científicos, incluido el correspondiente a la teología, quienes ayudaron a actualizar permanentemente dicha obra, teniendo en cuenta las diferentes etapas de aprendizaje. Entre dichos expertos, Kößling menciona a Johannes Wacker, quien creó diversas ediciones para los diferentes niveles educativos: «Im Auftrag seines Landesherrns arbeitete der Stuttgarter Schulmann Johannes Wacker sie (Gramatica D. Philipi latina cum exercitio) zum Schulbuch für den Gebrauch in einzelnen Stufen um» $»^{18}$ (Kößling, 1999: 80 $)^{19}$.

Por si fuera poco, la expansión del uso de dicha obra en instituciones educativas creció exponencialmente al convertirse Melanchthon en el referente principal que fue ya en el mundo académico de su época, razón por la que se le conoce como Praeceptor Germaniae. De hecho, dicha obra se benefició de su integración en el Programa escolar de 1528 concebido por el propio Melanchthon, tal como señala la valoración que de dicha expansión hace Kößling en el siguiente fragmento:

Als Lehrbuchautor wurde Melanchton wirksam durch sein bereit erwähntes Enchiridion, das Handbuch für den Anfangsunterricht. I 528 folgt seine Grammatica Latina, die sich gegen die Grammatik des Rivius behauptet, wohl auch, weil Melanchthon der Beschreibung der lateinischen Lektionenfolge in der Kursächsischen Schulordnung von 1528 deb Gang seines Buches zugrunde gelegt hat ${ }^{20}$ (Kößling, 1999: 96$)^{21}$.

La concepción reformadora que Melanchthon tuvo de la enseñanza, además de estar imbuida del espíritu humanístico, también se sustentaba en la idea de que esta debía preparar para lo que la Iglesia y el Estado exigen del joven habitante y del joven ciudadano, siguiendo la línea de la política educativa protestante alemana (Tröhler, 2013: 88) ${ }^{22}$, tal como Lutero y él mismo propusieron. Pero más aún,

16 «A modo de ejemplo, el horario escolar más antiguo que se conserva, el de la Nikoleischule del año I574, identifica la "Gramatica D. Philipi latina cum exercitio" como manual para las clases de lunes a viernes de 8-9 horas de tercer curso».

${ }_{17}$ Op. cit.

18 «Por encargo de las autoridades territoriales el pedagogo de Stuttgart, Johannes Wacker, trabajó en su reelaboración (Grammatica D. Philipi latina cum exercitio) para su uso en los diversos niveles».

19 Op. cit.

${ }_{20}$ "Como autor de manuales de clase, Melanchthon tuvo influencia a través del ya mencionado Enchiridium, pequeño manual de referencia para las clases en niveles iniciales. En i 525 le sigue su Gramática latina, que se impuso frente a la Gramática de Rivius debido a que fijó la sucesión de las lecciones de latín ubicándolas en cada uno de los momentos por los que transcurre su libro».

${ }_{21}$ Op. cit.

22 Tröhler, D.: Los lenguajes de la educación, Barcelona, Editorial Octaedro, 2013. 
dicha concepción no se agota en una forma teórica y abstracta de entenderla y formularla, sino que le dio una orientación práctica que desarrolló a lo largo de sus libros, utilizándolos como verdaderos apoyos de la clase de didáctica que se basaban en observaciones propias de su condición de docente, tal como lo señala el siguiente fragmento: «Melanchthon hat einen Großteil seiner wissenschaftlichen Arbeit der Erarbeitung von Lehrbücher gewindmet»"23 (Kößling, 1999: 74)24; este enfoque se constituyó en la base organizativa de la enseñanza en la nueva Iglesia protestante alemana de la que él era el máximo representante. Esta dimensión práctica y funcional explica, por una parte, el éxito de la ordenación escolar que puso en marcha y que materializó a través de los Reglamentos escolares; por otra parte, le hizo implicarse en experiencias educativas para poner en práctica formas diversas de aprendizaje. Un ejemplo de ello es la iniciativa que llevó a cabo con la creación de un pensionado -Schola domestica-(Faubell, 1984: 12I ${ }^{25}$ en su casa donde preparaba a jóvenes, futuros universitarios, en materias diversas como latín, griego, hebreo, matemáticas, ética y física aristotélica: «Melanchton hatte in Wittenberg einen engeren Kontakt mit studierenden angestrebt und I5I9 eine Schola domestica gegründet. Er möchte hoffen Talente zu fordern und zugleich eine Anregung zur Einrichtung ähnlicher Gemeinschaften zu geben ${ }^{26}$ (Kößling, 1999: 76) 27. $^{2}$.

La alta responsabilidad que Melanchthon tuvo en la naciente Iglesia y su gran preocupación por la enseñanza y su didáctica ${ }^{28}$ le llevaron a la elaboración de planes de estudio en escuelas y Universidades de algunos estados germanos, entre los cuales conviene mencionar la Ordenación Escolar para el Electorado de Sajonia (I528). Dichos planes fueron estructurados en clases de leer y escribir, gramática y composición constituyendo el latín la lengua articuladora de los mismos (Faubell, 1984: I2I-I22) ${ }^{29}$. Se trata de planes de estudio determinados por el dominio de los contenidos lingüísticos y no por la edad. En la práctica se quiere que jóvenes de diversas edades puedan experimentar la mejora conjunta de su formación lingüística, tal como se buscaba en la primera escuela (Schola domestica): «Nach Art der in den mittelalterlichen Bursen Wohn - und Lerngemeinschaften

\footnotetext{
${ }^{23}$ «Melanchthon ha dedicado la mayoría de su trabajo científico al desarrollo de los libros de texto».

24 Op. cit.

${ }_{25}$ Faubell, V.: «Melanchthon, Felipe (I497-1560)», en Escolano, A.: Historia de la Educación I, Madrid, Editorial Anaya, I984, pp. I2I-I22.

26 «Melanchthon se esforzó en Wittenberg por mantener un estrecho contacto con los estudiantes y en I5ı fundó una Schola domestica [...]. Con ella esperaba fomentar el talento y promover el establecimiento de comunidades similares».

27 Op. cit.

${ }_{28}$ La preocupación y el compromiso de Melanchthon de reformar la enseñanza en Alemania parten del conocimiento directo que tiene del sistema de enseñanza en los niveles no universitario y universitario. Conocimiento que le lleva a adoptar una actitud crítica ante la situación existente que describirá en su escrito «De Misseriis Paedagogorum». Cf. WOODward, W. H.: Studies in Education during the Age of Renaissance. 1400-1600, New York, Teachers College Press, Columbia University, 1967, p. 217.

29 Op. cit.
} 


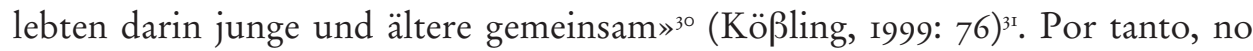
debe restringirse la influencia de Melanchthon a los primeros niveles educativos.

Aunque Melanchthon dedicó buena parte de su actividad a la organización de las escuelas, no obstante, la reforma de la universidad formó también parte de sus preocupaciones. Al tiempo que realizaba la reforma de la enseñanza secundaria, fue reclamado su asesoramiento por varias universidades (Tubinga, Leipzig, Heidelberg, Marburgo, Koenisberg y, cómo no, Wittenberg, su propia universidad, que se constituiría en el modelo de referencia para la reforma) que querían acometer su transformación. El intenso conocimiento que Melanchthon tenía del sistema universitario, por su condición de profesor del mismo, junto a su experiencia en la reforma de las escuelas, explica la fuerte demanda de asesoramiento que tuvo y la gran influencia de Melanchthon en dichas universidades alemanas:

In a large sense the university of Wittenberg became the model for the other Protestant universities... Königsberg was founded in 1544 almost exclusively according to directions given by Melanchthon, as was Jena in $1548 \ldots$ All the sciences, theologly, philology, law and medicine were studied in these universities according to Melanchthonian methods, with the Melanchthonian thoroughness and with the Melanchthonian view of honouring God and of carrying on an irrepressible conflict with an opposing ecclesiastical principle of higher education ${ }^{32}$ (Scheible, 1902: 139)33.

Los planes de estudio de Melanchthon proponen toda una diversidad de estrategias de aprendizaje que conduzcan al dominio lingüístico. A tal fin, indica que se debe partir de la lectura del Antiguo y del Nuevo Testamento y se deben hacer ejercicios de sintaxis y de gramática basados en dichos textos. Igualmente señala que debe profundizarse en la idea de que la gramática va de la mano de la retórica y la dialéctica. Consiguientemente no pueden descuidarse los ejercicios de estilo, de versificación y de diálogo. También presta especial atención a la fonética, a la forma de argumentar, así como a la cohesión y a la coherencia en los textos. Recomienda el estudio crítico de mucha y diversa literatura secundaria, como comenta el siguiente fragmento:

30 «A la manera de las residencias de estudiantes medievales, convivían en sus comunidades residenciales y de estudio alumnos de mayor edad con otros de menor edad».

31 Op. cit.

32 «En un sentido amplio, la Universidad de Wittenberg se convirtió en el modelo para las otras universidades protestantes... Königsberg fue fundada en I544, casi exclusivamente según las instrucciones dadas por Melanchthon, como Jena en I548... Todas las ciencias, teología, filología, derecho y medicina fueron estudiadas en estas universidades según los métodos de Melanchthon, con la minuciosidad de Melanchthon y con la visión de Melanchthon de honrar a Dios y de continuar un irrefrenable conflicto con el opuesto principio eclesiástico de la educación superior».

33 Scheible, H.: Melanchthon-Eine Biographie, München, Editorial C. H. Beck, 1997. Libro electrónico disponible en la web visitada por última vez el II de septiembre de 20Is: https://books. google.de/books?id=99KYr19xisAC\&printsec $=$ frontcover\&dq=Heinz + Scheible + Melanchthon $+B$ iographie\&source=bl\&ots=6cEWMZDKYI\&sig=JsNI3cXurGfu3aUe_RrsrChlfQA\&hl=de\#v=on epage $\& \mathrm{q} \& \mathrm{f}=$ false. 
Zur Schulug des Stils können kontroversthelolgische Themen gewählt werden, etwa die Widerlegung der Lehren der Wiederläufer und natürlich die Lektüre der lateinischer und griechischer Klassiker, auch Juristen und Philosophen. Auch Theologen sollten Philosophie studieren, diese aber von der Theologie deutlich unterscheiden ${ }^{34}$ (Scheible, 1997: 49-51 $)^{35}$.

Esta fue la reforma que Melanchthon llevó a cabo en las escuelas que fueron objeto de reforma o de nueva creación y en las que él intervenía directamente o indirectamente, como las de Wittenberg (I520), Eisleben (I525) y Núremberg (I524). En las tres escuelas, Melanchthon aplica el mismo modelo que busca conseguir una formación general y una instrucción intelectual, y ambas formaciones se transmiten teniendo en cuenta la vertiente de la educación religiosa. Las ligeras variantes que Melanchthon introduce en la aplicación del modelo de organización de la enseñanza responden a las particularidades que presenta cada una de las escuelas, que necesariamente tuvo que tener en cuenta para asegurar la viabilidad de la organización del sistema educativo (Labrador, 1984: 20-27) ${ }^{36}$.

Fruto, por una parte, de la experiencia que Melanchthon tiene de la organización de la enseñanza en las diferentes escuelas en las que elaboró sus planes de estudio y, por otra, del conocimiento que posee de la situación lamentable que padecen las escuelas alemanas son «Las Instrucciones a los visitadores (enviados) a los párrocos en el Principado de Sajonia» (I528), que redacta a petición del elector Juan Federico de Sajonia y bajo la recomendación de Lutero. En dicho documento Melanchthon manifiesta su filosofía educativa y señala las orientaciones que conviene seguir para el buen funcionamiento de las instituciones educativas y para solucionar lo que él califica «los muchos abusos en las escuelas infantiles» (Labrador, 1984: 33) 37 .

Algunas de las orientaciones que Melanchthon más incentiva para el buen funcionamiento de las escuelas son, por una parte, las visitas de inspección, ya que estas facilitarán el cumplimiento de los planes educativos: «Die bis zum Anfang des Dreißigjahrigen ziemlich regelmäßig durchgeführten Visitationen haben Anla $\beta$ geboten, Schulordnungen und Schulpläne im Laufe der Zeit sorgfältiger

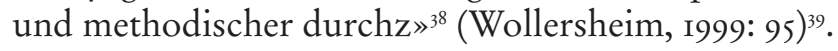

34 «Para el aprendizaje del estilo se pueden elegir temas de controversia teológica, como, por ejemplo, la oposición a las doctrinas de los rebautizados y naturalmente la lectura de los clásicos griegos y latinos, así como de juristas y filósofos. También los teólogos deberían cursar estudios universitarios de Filosofía y deberían distinguirlos claramente de la Teología».

35 Op. cit.

36 Op. cit.

37 Op. cit.

${ }_{38} \quad$ «Las visitas de la inspección realizadas con regularidad hasta el comienzo de la Guerra de los 30 años ofrecieron la oportunidad de implantar a lo largo del tiempo con mayor cuidado y de forma más metódica órdenes y planes escolares».

39 Wollersheim, W.: Philipp Melachthons Einflu $\beta$ auf das sächsische Schulwesen, en WarTeNBERG, G. y HEIN, M. (coords.): Editorial Evangelische Verlaganstalt, Universität Leipzig, I999. 
Pero, por otra parte, también Melanchthon considera importante el cumplimiento de la normativa educativa con carácter general, puesto que garantiza la unificación de la organización escolar y la efectividad del sistema educativo:

Die Durchsetzung und Einhaltung dieser Norm wird in zahlreichen Wiederholungsvisitationen immer wieder geprüft und eingemahnt. Das sich allmählich herausbildende relative einheitliche Niveau der Schulabsolventen ist für die Leistungsfähigkeit des Bildungssystems insgesamt auf Dauer gesehen ein villeicht noch wichtiger Fortschrit als der Siegeszug des Humanismus an den Schulen ${ }^{40}$ (Wollersheim, 1999: 94-95) ${ }^{4+}$.

Dichas orientaciones están referidas al conjunto de los elementos que conforman la organización de la enseñanza: profesores, padres, alumnos, contenidos, materiales, metodología (Melanchthon, 1853-60) ${ }^{42}$.

A los profesores, maestros de escuela, Melanchthon les exige dos cualidades que a lo largo del tiempo se han considerado como fundamentales en el oficio de maestro: tener experiencia en dicho oficio y estar dotado de la habilidad necesaria para desarrollar las tareas que conlleva. Ello requiere, en opinión de Melanchthon, que los maestros se preparen adecuadamente para el oficio que tienen encomendado de modo que posean las mismas cualidades que les exige a los predicadores: «Pues quien tiene que enseñar a otros debe tener un gran ejercicio y una especial habilidad, para obtener la cual hay que aprender durante mucho tiempo y desde la juventud» (Labrador, 1984: 32$)^{43}$.

Melanchthon tiene también muy en cuenta que la institución escolar es el espacio de aprendizaje para los alumnos, en el que los padres de los niños juegan un papel importante, tanto desde la perspectiva de la escolarización (número de alumnos que la frecuentan), como desde la motivación de los niños para ir a la escuela y el proceso de aprendizaje que tiene lugar en la misma. Lógicamente, en el contexto de principios del siglo XVI, lo que más importaba era motivar a los padres para llevar a sus hijos a la escuela ${ }^{44}$. Por ello, Melanchthon recomienda en sus Instrucciones que: «Los predicadores tienen que amonestar también a la gente de que sus hijos vayan a la escuela para que se instruya a la gente [...]» (Labrador, 1984: 32$)^{45}$.

40 «La implantación y el cumplimiento de esa norma se comprobará y reivindicará a través de numerosas y repetidas visitas de inspectores. Visto en el largo plazo, la progresiva formación de un nivel relativamente unificado entre aquellos que han superado la escuela es, en su conjunto, un avance para la efectividad del sistema educativo de las escuelas, quizá aún más importante que el triunfo del Humanismo».

${ }^{41}$ Op. cit.

${ }_{42}$ Melanchthon, Ph.: Corpus Reformatorum Philippi Melanchthonis Opera Quae Supersunt Omnia (CR) (edit.), C. B. Bretscneider and H. E. Bindseil, 28 vols. Halle I834-52, Brunswick, I853-60.

43 Op. cit.

${ }_{44}$ Conviene recordar que la tasa media de escolarización en Europa, incluida Alemania, a mediados del siglo XV no llegaba al 20\%. Cf. Roser, M.: Literacy rates in Western Europe from the ts $^{\text {th }}$ century to now. Published online at OurWorldInData.org. 2015, Retrieved from: http://ourworldindata.org/data/education-knowledge/literacy/ [Online Resource].

${ }_{45}$ Op. cit. 
Los contenidos a enseñar y los materiales a utilizar que Melanchthon propone en los planes de estudio, cuyas líneas directrices explicita en las Instrucciones, se caracterizan fundamentalmente por primar el uso del latín, por simplificar los materiales de clase y por hacer una enseñanza progresiva dividiendo a los niños en grupos. Así expresa Melanchthon dichas orientaciones:

En primer lugar, los maestros de escuela deben tener en cuenta enseñar a los niños solamente en latín, no en alemán o griego o hebreo, como muchos han hecho hasta ahora, que abruman a los pobres niños con tal multiplicidad de cosas, que no solamente es infructuosa, sino dañina. Se ve también que semejantes maestros no piensan en el provecho de los niños, sino que toman tantas lenguas buscando su propia honra. En segundo lugar, no deben recargar a los niños con muchos libros, sino en todos sus procedimientos huir de la cantidad. Y en tercer lugar, es necesario que dividan a los niños en grupos (Labrador, 1984: 33$)^{46}$.

De estas tres directrices u orientaciones, la tercera (dividir a los niños en grupos) es la que Melanchthon desarrolla con más detalle. Al respecto, el proceso de aprendizaje de los niños lo divide en tres niveles o grupos, estableciendo entre ellos su progresividad en términos de objetivos. De este modo, el primer grupo es el básico y tiene como objetivo principal aprender a leer; el segundo grupo es el intermedio y tiene como objetivo principal aprender la Gramática, una vez que ya saben leer; y el tercer grupo es el nivel superior y está formado por los alumnos más adelantados y tienen como objetivo principal de aprendizaje familiarizarse con la Dialéctica y la Retórica, una vez que dominan bien la Gramática (Labrador, 1984: 32-3647. La metodología que Melanchthon propone para cada uno de estos cursos o etapas es una de las contribuciones más relevantes que Melanchthon nos ofrece desde la perspectiva organizativa y didáctica. Un buen ejemplo de su sentido organizativo y didáctico es la defensa que Melanchthon hace de la elaboración individualizada de los planes de estudio y de la planificación de los horarios en el ámbito universitario: «Die von Melanchthon in seinem ersten Rektorat erlassenen Atudienordnung sieht vor, da $\beta$ für jeden neuen Studenten ein individueller Stundenplan erstellt wird» $\star^{8}$ (Scheible, 1997: 49-51 $)^{49}$.

No cabe duda de que el desarrollo y la estructuración que hace Melanchthon del qué y del cómo enseñar se apoya en tres ejes significativos que ponen de manifiesto su gran capacidad pedagógica (Hofmann: 1963$)^{\text {s。: }}$ el uso amplio de los saberes, la agrupación de los alumnos (3 grupos) según su nivel de conocimientos en busca de la máxima eficacia en la enseñanza, y un claro carácter práctico en su estrategia docente, que le llevó a proponer el uso de métodos y técnicas didácticas

${ }^{46}$ Op. cit.

47 Op. cit.

${ }_{48}$ «En la orden que emitió durante su primer rectorado está previsto que se confeccione un horario individual para cada estudiante».

49 Op. cit.

so Hofmann, F.: «Philipp Melanchthon und die zentralen Bildungsprobleme des Reformationsjahrhunderts», en Ph. Melanchthon. Humanist, Reformator, Praeceptor Germaniae, Berlin, Akademie. Verlag, 1963, pp. 83-I09. 
como el debate y la declamación (Stupperich, 1960: 56) (1 $^{\mathrm{s}}$ con la intención de facilitar el aprendizaje. Dichos ejes conductores los orientó a:

I. Fundamentar la acción educativa sobre una base antropológica. El hombre para Melanchthon constituye el principio y el fin de la educación. Ello explica su apuesta permanente, a pesar de las dificultades que fueron surgiendo, por el ideal humanista.

2. Crear un modelo de escuela, la escuela protestante, de espíritu humanista y basada en el conocimiento universal y enciclopédico. Coherente con esta visión, Melanchthon incluye en el currículum escolar la presencia de varias materias pertenecientes a saberes diversos e interconectados por su visión filosófica y teológica. La perspectiva filosófica de Melanchthon guarda relación con su condición de filólogo y reformador (Kusukawa, 1999: xii) ${ }^{52}$. Igualmente, su perspectiva teológica le lleva entender que el saber no es un fin en sí mismo, sino que debe estar al servicio del conocimiento de Dios.

3. Organizar el sistema escolar, tanto el no universitario -a través de la nueva orientación de las escuelas como de la creación de nuevos centros escolares-, como el universitario (Kusukawa, 1999: xi) s3, al que le dio la máxima prioridad, al pensar que la buena educación de los jóvenes era un capital importante para la Nación y los Estados. La organización escolar de la reforma protestante se caracterizó, siguiendo el pensamiento de Melanchthon y Lutero, por dos aspectos que suponían una ruptura con la tradición anterior: por una parte, por limitar los contenidos de la enseñanza a lo que ellos consideraban lo esencial (Martín, 2010: 222) ${ }^{54}$, eliminando la diversidad superflua existente hasta el momento al no facilitar el aprendizaje, sino más bien impedirlo; y, por otra parte, por trasladar la responsabilidad de la organización escolar tanto a la nueva Iglesia como al Estado.

Melanchthon no se limita al abordar la cuestión educativa a establecer principios generales de política educativa y de organización escolar. Va más allá, y se adentra en aspectos prácticos y funcionales que, al límite, garantizan el buen funcionamiento del sistema educativo, sea simplemente el escolar como el universitario. Al respecto, reflexiona y aporta contribuciones relativas a aspectos que considera relevantes para la organización escolar, tales como el orden a seguir en el aprendizaje y el papel de las escuelas. En ambos casos, Melanchthon utiliza los textos de los discursos pronunciados por Gaspar Crucigerss, por un lado, y por Bernard Ziegler ${ }^{56}$, por otro.

sI Stupperich, R.: Melanchthon, Berlin, Gruyter, i960.

52 Kusukawa, S.: «Introduction», en Orations on Philosophy and Education, Edited by Shachiko Kusukawa: translated by Christine F. Salazar, Cambridge, Cambridge University Press, 1999, pp. XI-XXXVI.

53 Op. cit.

${ }_{54}$ Martín, M. A.: «Implicaciones educativas de la Reforma y Contrarreforma en la Europa del Renacimiento», Revista Cauriensia, vol. v (2010), pp. 215-236.

"Gaspar Cruciger (I504-48) fue decano de la Facultad de Letras y el discurso que pronunció en la Graduación de Másters del año I53r versó sobre el orden del aprendizaje.

${ }_{56}$ Bernard Ziegler (1496-1556) fue profesor de Hebreo en la Universidad de Leipzig en la que pronunció el discurso que versó sobre el papel de las escuelas. 
Respecto al orden a seguir en el aprendizaje, el referido texto dirigido a los jóvenes señala la importancia de empezarlo conociendo los elementos de la filosofía, y a partir de ellos avanzar en el de las disciplinas fundamentales, entre las que se encuentran las Letras. Este orden de aprendizaje asegura de entrada la consecución de la mitad del conocimiento previsto. La filosofía, en este orden lógico, se constituye en el primer objetivo a conocer, y a partir de la misma se accede al conocimiento de las otras disciplinas (Melanchthon, 1999: $8^{57}$ y Melanchthon, I834-52 y 1853-60 8 ).

Al hablar del papel de las escuelas, se recuerda la relación histórica estrecha entre la escuela y la Iglesia, razón por la que los gobernantes deben hacer que las universidades cumplan su cometido científico y religioso mediante las orientaciones pertinentes y la preparación adecuada de los profesores que deben ser «intelligent, erudite and distinguished by virtue and sagacity, who both understand the method useful for rethoric and perform their duty faithfully»59 (Melanchthon, 1999: 15) ${ }^{60}$. Precisamente, para que los profesores cumplan su cometido deben ser retribuidos decentemente, pero evitando, al mismo tiempo, salarios excesivos que impidan el cumplimiento de sus tareas: «The faithful teachers should also be given decent wages. Excessive opulence is not to be desired, because the attachment to wealth obstructs studying and fosters extravagant living in the idle» ${ }^{61}$ (Melanchthon, 1999: 16) ${ }^{62}$.

En definitiva, la filosofía de la educación que Melanchthon impulsa en la Alemania de la Reforma a través de la organización de la enseñanza, tanto en las escuelas como en las universidades, condujo a que los individuos aprendieran a articular sus pensamientos y sentimientos, sus experiencias de conocimiento científico y todo lo que les motivaba y preocupaba; es decir, a saber establecer la adecuada relación entre ciencia, cultura, religión y sociedad. Sin esta filosofía y perspectiva, hubiera sido difícil de explicar la emergencia de relevantes figuras pertenecientes a distintas áreas de conocimiento que destacaron por su gran dominio de la lengua y por su deseo de unificar religión y cultura: «Without these universities German Theology wouldn't have a Gerhard, a Spener, a Schleiermacher, a Dorner, nor German Philosophy, a Leibnitz, Kant, Hegel, a Lotze, nor German Poetry, a Gellert, a Klopstok, a Goethe, a Schiller» ${ }^{63}$ (Richard, 1902: 139-I40) ${ }^{64}$.

57 Melanchthon, Ph.: Orations on Philosophy and Education, Edited by Shachiko Kusukawa: translated by C. F. Salazar, Cambridge, Cambridge University Press, 1999.

s8 Op. cit.

59 «Inteligentes, eruditos y distinguidos por la virtud y la sagacidad que, además de entender sobre el método útil para la retórica, cumplen fielmente con su deber».

60 Op. cit.

${ }_{61}^{61}$ «Los fieles maestros también deberían recibir salarios decentes. Opulencia excesiva no es deseable, porque el apego a la riqueza impide estudiar y fomenta una vida extravagante y ociosa».

62 Op. cit.

${ }_{3}$ «Sin estas universidades la teología alemana no habría tenido a un Gerhard, a un Spener, a un Schleiermacher, a un Dorner; ni la filosofía alemana habría tenido a un Leibnitz, a un Kant, a un Hegel, a un Lotze; ni la poesía alemana habría tenido a un Gellert, a un Klopstok, a un Goethe, a un Schiller».

${ }_{64}$ Richard, J. W.: Philip Melanchthon, the Protestant preceptor of Germany, I497-1560, New York/London, (edit.) G.P. Putnams' sons, 1902. Libro electrónico disponible en la web visitada por última vez el II de septiembre de 20I5: https://ia80o30I.us.archive.org/8/items/philipmelanchthooorichrich/philipmelanchthooorichrich.pdf. 
Las importantes y variadas actuaciones de Melanchthon en el campo educativo le valieron el nombre de Praeceptor Germaniae, calificativo con el que se le conoce no solo en Alemania, sino también en todo el mundo. Tanto los príncipes electores como el incipiente movimiento religioso luterano supieron valorarle su labor y su gran contribución al campo de la enseñanza. Todo ello justifica, en opinión de Kusukawa, el calificativo de Praeceptor Germaniae, tal como nos lo señala en el siguiente fragmento:

He reformed the arts curriculum of the University of Wittenberg, and revived classical learning in schools and universities across Germany. He wrote textbooks on most of the subjects in the arts curriculum: Latin and Greek grammar, rethoric, dialectics, moral philosophy and natural philosophy. For subjects on which he did not write, such as geometry, arithmetic, astronomy and astrology, he wrote commendatory prefaces to textbooks by others. For classroom use, he edited and commented upon texts of numerous classical authors such as Cicero, Demosthenes, Virgil, Ovid, Hesiod and Ptolemy. These texts and textbooks were used well into the seventeenth century, and he trained and inspired a vast number of students who in their turn passed on the message of their teacher to the next generation. For his educational reforms, Melanchthon was accorded the title of Praeceptor Germaniae, the teacher of Germany ${ }^{65}$ (Kusukawa, 1999: xi) ${ }^{66}$.

\section{La contribución de Melanchthon a la lengua}

Aunque el uso del buen latín constituía para Melanchthon un objetivo principal, dado que era un buen humanista, así como catedrático de Griego en la Universidad de Wittenberg, sin embargo, tuvo también una actitud positiva hacia el uso del alemán y hacia las lenguas vernáculas en general, ya que contribuían al conocimiento y al desarrollo de los saberes.

Todo lo que Melanchthon pensaba respecto a las lenguas y a su uso concreto era coincidente con lo que Lutero escribió al respecto en varios de sus escritos, cuyo pensamiento expresó, muy especialmente, en su obra -anteriormente mencionada-A los magistrados de todas las ciudades alemanas, para que construyan y mantengan escuelas cristianas, obra de carácter pedagógico que escribió en I524.

65 «Melanchthon reformó el currículo de las Artes en la Universidad de Wittenberg y activó la enseñanza clásica en escuelas y universidades de toda Alemania. Escribió libros de texto en la mayoría de las materias del currículo de las Artes: gramática de latín y griego, retórica, dialéctica, filosofía moral y filosofía natural. En las materias en las que él no escribió, como la geometría, aritmética, astronomía y astrología, escribió elogiosos prefacios a libros de texto escritos por otros. Para el uso de la clase, editó y comentó textos de numerosos autores clásicos como Cicerón, Demóstenes, Virgilio, Ovidio, Hesíodo y Ptolomeo. Estos textos y manuales fueron utilizados en el siglo XVII, y formó e inspiró a un gran número de estudiantes que a su vez pasaron el mensaje de su maestro a la siguiente generación. Debido a sus reformas educativas, a Melanchthon le fue concedido el título de Praeceptor Germaniae, el maestro de Alemania».

${ }_{6}^{6}$ Op. cit. 
La preocupación de Melanchthon por las lenguas no es solo teórica. Al contrario, como responsable de la organización de la enseñanza en la Reforma, se preocupa de que las lenguas ocupen el espacio que les debe corresponder en la nueva enseñanza de la Reforma en Alemania, a través de los nuevos planes de estudio a implantar. Es consciente de que la debilidad del sistema de enseñanza en Alemania es la causa de las deficiencias en el conocimiento de las lenguas en el país. Por ello, la reforma del sistema de enseñanza que lidera, tanto el universitario como el no universitario, supondrá un impulso en la mejora de su aprendizaje, a través del uso oral de las mismas, de las técnicas del diálogo, de las disputas y las declamaciones. Los campos de la filología (que constituyen el territorio de las lenguas) y de la filosofía son, en opinión de Melanchthon, los que fundamentan el conjunto de los saberes, incluido el teológico, como nos lo recuerda el siguiente fragmento: «In the prominence given to these literary and philosophical studies, we have an illustration of Melanchthon's fundamental principle, viz. that all thorough training in theology must rest on a philological and a philosophical foundation $\gg^{67}$ (Richard, 1902: 138$)^{68}$.

Pero la preocupación fundamental de Melanchthon por mejorar el aprendizaje de las lenguas se focaliza, básicamente, en las lenguas clásicas, especialmente en el latín, pero sin que esto suponga darle la espalda a las lenguas vernáculas, en este caso al alemán. Por ello, utilizó la lengua alemana para explicar el significado de las palabras y cada una de las particularidades lingüísticas de la lengua latina, tal como hizo en su obra Exercitium puerorum Gramaticale per dietas distributum $(\mathrm{I} 485)^{69}$. En esto Melanchthon no se aleja de la opinión de Erasmo, o incluso de Juan Luis Vives, que aunque usaron también la lengua latina en exclusiva en sus escritos, sin embargo, cohabitaron razonablemente con las lenguas vernáculas.

El pensamiento de Melanchthon acerca de la lengua y de las lenguas no se reduce a mostrar su importancia y relevancia. Va más lejos, ya que le preocupa mucho el uso didáctico de las mismas, tal como anteriormente se ha señalado. Por ello considera que las gramáticas excesivamente teóricas y extensas no favorecen la práctica y son negativas desde un punto de vista didáctico. De ahí la crítica que hizo a la metodología seguida por el Doctrinale de Villa Dei y la renuncia a hacer definiciones demasiado complejas. Su estrategia didáctica de suplir la abstracción con la capacidad deductiva del alumno le permite introducir ejemplos de autores de la Antigüedad como Terencio, Cicerón o Virgilio. De hecho, su gramática estaba

${ }_{67}$ «En el protagonismo dado a los estudios literarios y filosóficos, tenemos una explicación del principio fundamental de Melanchthon, es decir, que toda la formación en teología debe apoyarse en una base filológica y filosófica».

68 Op. cit.

69 Esta obra fue impresa, por primera vez, en Amberes (I488). En el prólogo a la edición de

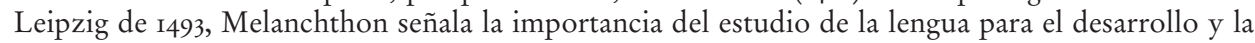
transmisión del conocimiento: «Quis, oro, sine grammatica sperandus est poeta, quis causarum orator, quis contra haereticos valens disputator. Aut quis unquam alteri deditus studio sine grammatica fuerit?». Traducción del autor: «¿Quién, pregunto, podría sin la gramática ser poeta, quién defensor de causas, quién argumentar con fuerza contra los herejes? O ¿quién se ha podido dedicar alguna vez a cualquier otro estudio sin la gramática?» (KöBLING: 1999, op. cit.). 
orientada a su uso práctico en la clase de latín, en la que los estudiantes hacían la lectura de dichos textos y se les inculcaba el uso oral y escrito de la lengua.

El propio pensamiento de Lutero sobre la reforma de la enseñanza es básicamente el pensamiento que Melanchthon traslada a la organización de la enseñanza en la Reforma en la que siempre acentuó su orientación didáctica y en la que incorporó, como se ha dicho anteriormente, aquellos nuevos aspectos que resultaban relevantes para el aprendizaje de la juventud, entre los que se encuentra el aprendizaje y uso de las lenguas, no solo clásicas, sino también vernáculas.

No cabe duda de que la preocupación de Melanchthon por los aspectos didácticos y pedagógicos en la enseñanza alimentó el propio pensamiento de Lutero al respecto e hizo que en este campo la sintonía entre Melanchthon y Lutero fuera total. Tal hecho explica los resultados positivos obtenidos por la Reforma en materia de educación en aquellas ciudades alemanas donde la nueva enseñanza se organizaba. Con el tiempo, la labor didáctica de Melanchthon ha sido reconocida, considerando su obra reformadora como un activo importante en la tradición de la pedagogía alemana, tal como lo señala el siguiente fragmento: «La historia de la Pedagogía ha hecho justicia a las iniciativas de Lutero, secundadas, precedidas y perfeccionadas por las del colaborador y excepcional pedagogo Melanchthon» (Egido, 2006: 27) ${ }^{70}$.

\section{Melanchthon en medio de Erasmo y de Lutero, o entre la cultura humanista europea y la Reforma alemana}

La doble faceta de Melanchthon, humanista y reformador, le situó en relación y bajo la influencia de dos personajes relevantes y decisivos en la cultura de su tiempo como Erasmo y Lutero (Alcalá, I97I: 12 ) $^{7 \mathrm{r}}$; el primero, desde la perspectiva humanista (Erasmo, 1971: 454-455 $5^{72}$. Halkin, 1971: 89-9073 y Febvre, 1970: 9374), y el segundo, desde la perspectiva de la Reforma. Es importante subrayar, en este contexto, que Erasmo y Lutero son hombres de la misma época -temporal y culturalmente hablando-, que compartieron inicialmente las mismas ansias de reforma que caracterizaron a su tiempo (Eckermann, 2005: 623-624) ${ }^{75}$, es decir, el Humanismo renacentista. Ambas perspectivas relacionales contribuyen al conocimiento de Melanchthon. Entre estos tres personajes, Melanchthon, Erasmo y Lutero, fluía una energía especial que ponía de manifiesto el aprecio y valoración

70 Egido, T. (ed.): Lutero. Obras, Salamanca, Ediciones Sígueme, 2006.

${ }^{71}$ AlcalÁ, A.: «Introducción», en Servet, Miguel: Treinta cartas a Calvino. Sesenta signos del Anticristo. Apología de Melanchthon, Madrid, Editorial Castalia, 1971, pp. 9-72.

${ }_{72}$ ERASMO DE ROTTERDAM: La paráclesis o exhortación al estudio de las letras divinas. Edición y prólogo de Dámaso Alonso, Reimpresión facsimilar, Madrid, Consejo Superior de Investigaciones Científicas (CSIC), I97I.

73 Halkin, L.-E.: Erasmo, México, Fondo de Cultura Económica, I97I.

74 Febvre, L.: Erasmo, la Contrarreforma y el espíritu moderno, Barcelona, Martínez Roca, 1970.

75 Eckermann, J. P.: Conversaciones con Goethe, Barcelona, Acantilado, 2005. 
que se tenían (Nisard, 2010: $56^{76}$ y Lutero, 2006: $378-379^{77}$ ), pese a las discrepancias existentes entre Erasmo y Lutero (Bello, I983: 50)78. A lo largo de sus prolongadas relaciones, Melanchthon actuó siempre de bisagra, facilitando la relación o intentando evitar la ruptura que finalmente se produjo entre Erasmo (Febvre, 1956: I46-I47) ${ }^{79}$ y Lutero (Nisard, 2010: 70 y 76$)^{80}$.

El conocimiento de Melanchthon se enriquece cuando se le pone en relación con Erasmo y con Lutero. Las perspectivas humanistas y de la religión de ambos (Egido, 2006: 28-29) $)^{8 \mathrm{r}}$ no cabe duda de que constituyeron puntos de referencia para Melanchthon. Sin embargo, la vinculación de Melanchton con Lutero adquiere una especial significación en su vida, razón por la que nos ocupamos de ello a continuación.

\section{I. Melanchthon y Lutero}

Resulta difícil analizar el perfil humanista de Lutero y no establecer su relación con Melanchthon. El destino hizo que estas dos grandes figuras de la Reforma protestante alemana haya que asociarlas, puesto que las trayectorias de sus vidas y obras fueron básicamente en direcciones muy cercanas, aunque no idénticas, puesto que hay aspectos diferenciales entre ambos.

En la vida de Melanchthon (I497-1560) hay, pues, un antes y un después del encuentro con Lutero en Wittenberg (I528), este ocupando la cátedra de Teología bíblica de dicha universidad y Melanchthon la cátedra de Griego. Anteriormente a este momento, sus vidas discurrieron por caminos y lugares distintos.

La cátedra de Griego en Wittenberg dio a Melanchthon la oportunidad de entrar en contacto con la reforma religiosa que se estaba iniciando y, cómo no, de conocer y entablar relación con su líder natural, Martín Lutero. Ambos, colegas de la misma universidad, también tenían en común bastantes ideas, valoraciones y percepciones, tanto de la situación de los saberes científicos y religiosos de aquel momento como del estado de la Iglesia.

Bien pronto, las relaciones y colaboraciones entre Lutero y Melanchthon se estrecharon, a pesar de tener caracteres y sensibilidades diferentes. Ello hizo, por una parte, que Melanchthon se iniciara en los estudios de Teología de la mano de Lutero, obteniendo finalmente el título de Bachiller de estudios bíblicos que le capacitaba para impartir lecciones sobre la Biblia, llegando el propio Lutero a reconocer la competencia que había adquirido en estos estudios; por otra parte, también Lutero

7 Nisard, D.: Erasmo, Tomás Moro, Melanchthon, Sevilla, Editorial Trifaldi, 20 o.

77 Lutero, M.: Obras. Edición preparada por T. Egido, Salamanca, Ediciones Sígueme, 2006.

${ }_{78}$ BeLLo, E.: «En el origen de la modernidad, la libertad: Giovanni Pico y Lutero», en BeLLo, E. (ed.): Filosofía, sociedad e incomunicación. Homenaje a Antonio García Martínez, Murcia, Universidad de Murcia, 1983, pp. 33-57.

79 Febvre, L.: Martín Lutero. Un destino, México, Fondo de Cultura Económica, 1956.

8o Op. cit.

81 Op. cit. 
encontró en Melanchthon un buen colaborador en la empresa de reforma en la que estaba implicado, concretamente en las traducciones del Nuevo Testamento (I522) y del Antiguo (1534), gracias a su conocimiento de las lenguas clásicas.

La colaboración entre Lutero y Melanchthon fue más allá de lo puramente académico y profesional, ya que este fue asumiendo progresivamente responsabilidades en la reforma religiosa impulsada por Lutero. Pese a ciertas discrepancias entre ambos, la colaboración fue permanente y sólida hasta que sobrevino la muerte a Lutero. Hasta tal punto Melanchthon se ganó su confianza y la del propio entorno de este, que se vio empujado a tomar las riendas de la organización de la Iglesia reformada, una vez acaecida su muerte.

Dos aspectos, a nuestro entender, merecen la pena ser subrayados, ya que marcan, por un lado, la diferencia entre estas dos figuras dominantes del reformismo y, por otro, la distribución de tareas que cada uno de ellos asume en la organización institucional de la naciente Iglesia reformada.

El primero de estos aspectos es la concepción humanista que uno y otro tienen. Melanchthon, a diferencia de Lutero, situó al hombre en el centro de su visión filosófica, defendiendo sus potencialidades en relación con la mejora y progreso del mundo y de la ciencia. Esta visión le hizo compartir los ideales humanistas de su tiempo (Alcalá, I98I: I2) ${ }^{82}$.

Es cierto que esta visión antropológicamente optimista de Melanchthon se vió rebajada cuando tuvo que compatibilizarla con la que tenía al respecto Lutero y con el credo protestante, tal como puso de manifiesto en sus Principios básicos de teología o hipótesis teológicas (I52I). Sin embargo, Melanchthon, por formación y pragmatismo, pese a verse condicionado por los principios que animaban la Reforma, logró escapar del radicalismo de Lutero en esta cuestión, admitiendo la autonomía del hombre en los asuntos terrenales, pese a considerar a Dios como la única fuente de salvación (Pena, 2002: Io) ${ }^{83}$.

Melanchthon en la visión que tiene del hombre se aleja suficientemente de la postura de Lutero, puesto que tanto antes de conocerlo en Wittenberg (en esta época compartía totalmente los ideales humanistas del Renacimiento [Vergara, 20I2:25] $)^{84}$, como después (distinguía la autonomía divina y humana según se tratara de asuntos salvíficos o puramente terrenales), logró asignar al hombre una autonomía en su voluntad de actuar en el mundo. Esta visión no era compartida por Lutero, quien vio al hombre como siervo de Dios, vaciándolo de toda autonomía. Lógicamente, esta diferencia de opinión entre ambos en esta materia no pasó de «amigable disentimiento» (Egido, 2006: 4I ${ }^{85}$, o sirvió «para frenar el antihumanismo de Lutero» (Esteban, 1997: 34) ${ }^{86}$.

82 Op. cit.

${ }_{83}$ Op. cit.

${ }_{4}$ Vergara, J.: «Presentación. Humanismo y renovación educativa: una mayéutica para el hombre occidental», Historia de la Educación, vol. 3I (20I2), pp. 23-32.

${ }^{8}$ Op. cit.

86 Esteban, L. (ed.): Cuatro estudios a una obra o el «Arte de Enseñar» de Juan Luis Vives, Valencia, Ayuntament de Valencia, 1997. 
El segundo aspecto que merece ser examinado y que ayuda a ver la función de complementariedad que Melanchthon y Lutero desempeñaron entre sí, se refiere al papel que jugó el primero en la organización escolar que tanto ansiaba Lutero. Este, con la implantación del luteranismo, buscó la desaparición de las redes tradicionales (conventos, cabildos, etc.) que aseguraban la enseñanza de niños y jóvenes y trasladó dicha responsabilidad a la autoridad secular. Pero a este deseo y voluntad había que darle forma, mediante una nueva organización escolar. Nadie mejor que Melanchthon para llevar a cabo este proyecto y al que dedicó buena parte de su tiempo, tanto durante la vida de Lutero como después de su muerte.

En este proyecto educativo, Lutero y Melancthon jugaron un papel importante, cada uno con una función distinta. Lutero como inspirador del mismo y Melanchthon como el ejecutor. Curiosamente, la visión que ambos tienen del objetivo y organización de la reforma escolar es coincidente: una visión humanista; lo importante es organizar una educación que llegue al pueblo, que se dé una cultura general que permita conocer la antigüedad clásica, que favorezca el conocimiento de las lenguas clásicas, principalmente el latín, que se ayude en el aprendizaje usando la lengua propia, en este caso el alemán (Monreal, 20I2: $197)^{87}$, que se enseñen diversidad de disciplinas, como las artes, la historia, la música, las matemáticas, etc. Lutero, en este campo de la educación, por puro pragmatismo muestra su cara de humanista, una vez que se ha distanciado de los principios que orientan la cuestión teológica, tal como la plantea. Por ello, en este proyecto todo es coincidencia con Melanchthon (Egido, 2006: 27) ) $^{88}$.

Melanchthon desarrollará, pues, en base al espíritu humanista y con el asentimiento de Lutero, la Ratio Studiorum protestante, es decir, la organización escolar protestante $^{8}$. Su propuesta se adelantará en el tiempo a lo que Juan Luis Vives propondrá en su obra De Disciplinis, en la que, sin lugar a duda, muestra su influencia; igualmente se anticipará a otros reconocidos pedagogos como Sturm (I507-I589), amigo de Erasmo y Lutero y notable pedagogo de la Reforma, y Comenio (I5921670), filósofo, teólogo y pedagogo, a quien se considera el padre de la Pedagogía. En este contexto histórico y humanístico renacentista hay que situar también la Ratio Studiorum de I599 de la Compañia de Jesús (Labrador, 1999: II8) ${ }^{90}$ que comparte elementos comunes, desde la perspectiva de la reforma educativa católica, con la Ratio Studiorum de la reforma protestante (Labrador, 2013: 74)9.'.

Felipe Melanchthon, gran humanista y fiel discípulo de Lutero, respondió también a las expectativas que los humanistas alemanes pusieron en Lutero

${ }^{87}$ Monreal, J. L.: «La perspectiva religiosa y el uso de la lengua en Lutero», Revista FUTHARK, vol. 7 (2012), pp. 199-227.

${ }_{88}$ Op. cit.

89 Melanchthon estructura la organización escolar en tres niveles para los niños: Elemental, Gramatical y Retórica y Dialéctica. Para los jóvenes, Melanchthon crea el Gimnasio como institución de la segunda enseñanza.

90 LABRADOR, C.: «La Ratio Studiorum de I599. Un sistema educativo singular», Revista de Educación, n. ${ }^{\circ} 319$ (1999), pp. II7-I34.

91 Labrador, C.: «La Ratio Studiorum de i599: Un modo de ser y hacer en educación», Signos Universitarios. Revista de la Universidad del Salvador, año 32, n. ${ }^{\circ} 9$ (2013), pp. 69-93. 
(Monreal, 2012: 193 ${ }^{92}$ al proponer con claridad cómo entendía que debía ser, por ejemplo, la nueva orientación de la enseñanza en el nuevo contexto histórico del Humanismo renacentista:

En una oración pronunciada en Wittenberg en IsI8 sobre: ¿Cómo se han de corregir los estudios de los jóvenes?, exigía urgentemente el estudio del griego, que se debía unir con el latín para que penetraran hasta la misma médula en cada escritor, ya sea filósofo, teólogo, historiador, orador o poeta y a fin de que no se abracen solamente sus sombras. «Tened el valor de la prudencia. Cultivad los latinos, entregaos al griego, sin el cual el latín no se puede cultivar en realidad». Sin conocimiento del griego y el hebreo nadie se puede aventurar en los estudios teológicos. Aún el estudio de la historia es imprescindible (Jansen, r925: Parte Segunda, 176)93.

Casi cinco siglos después de la muerte de Melanchthon, todavía se hacen balances de su vida y obra que no hacen sino resaltar la relevancia de su contribución en diferentes aspectos, marcados siempre por el espíritu humanista, aunque estos estuvieron bastante oscurecidos por la envolvente figura de Lutero.

En resumen, se puede indicar que la posición de Melanchthon respecto a Erasmo y Lutero en cuanto a las temáticas principales que les relacionaron fue siempre de cierto equilibrio y de cierta equidistancia; hecho que explica haber compartido un territorio común, desde la cercanía, tanto con uno como con otro. Las coincidencias y divergencias que Melanchthon manifiesta respecto a ambos en determinadas cuestiones responden siempre a su profundo espíritu humanista, a su vivencia de la cultura europea y a su compromiso con las reformas tanto internas a la Iglesia como a la sociedad del momento.

\section{Conclusión: Melanchthon, reformador de las letras y de la religión}

Melanchthon fue un reformador, un verdadero humanista renacentista. La doble faceta en la que su vida se expresa, las letras y la religión, respiran aires de reforma. El territorio de las Letras lo transita, en las diversas expresiones de los saberes, de manera innovadora, abierta e interdependiente. El acceso a las lenguas permite el acceso a los saberes, sin excluirse en dicho cometido unas lenguas respecto a otras: las clásicas no niegan las vernáculas, sino que las enriquecen. Por otra parte, el territorio de la religión está también para Melanchthon necesitado de reforma. La energía que genera en Melanchthon su espíritu renovador humanista invade también su universo religioso, sometiéndolo igualmente a un proceso de reforma que se materializa con su acercamiento a Lutero y a todo lo que él representa. Esta es la motivación que orienta la vida, el pensamiento y la trayectoria de Melanchthon.

92 Op. cit.

93 Janssen, J.: «La cultura alemana antes y después de Lutero», Historia Universal, vol. viII bis, Barcelona, Librería Religiosa, I925. 
Si es relevante la actitud reformadora de Melanchthon en los campos de las letras y de la religión, no lo es menos su forma de actuar y el modo de orientar su comportamiento, que ciertamente tiene mucho que ver con lo más auténtico del humanismo renacentista: pasión por el conocimiento, hacerlo accesible, saberlo comunicar y hacer que sea un factor de cambio y de reforma.

Tras una trayectoria académica y científica precoz y brillante, el reto principal de Melanchthon fue el de saber transmitir y comunicar el conocimiento necesario para contribuir a las reformas de las letras y de la religión, huyendo del ensimismamiento, de la especulación, de la retórica y del dogmatismo, y solo buscando ser sincero y honesto con la gente para la que escribe, tal como lo afirma en varios de sus escritos:

Tan solo he aplicado -dice en alguna parte- todas las fuerzas que mi espíritu me permitía y todos los esfuerzos de mi voluntad a explicar con claridad y precisión tan grandes materias, y a dar a la juventud opiniones rectas y moderadas. En la medida en que me conozco, afirmo veraz y conscientemente, que no he tenido jamás otra intención que la de servir al público (Epist., IX, Libro II, en: Nisard, 20I0: 373)94.

En la forma de orientar la comunicación de los saberes para hacerlos accesibles al público, Melanchthon se guía por el método puesto en práctica por los clásicos antiguos (Labrador, 1984: 31 I5. Su referencia metodológica, como señala Nisard, la toma de la tradición humanista renacentista:

El arte de los antiguos no es más que el conocimiento de los caminos más seguros y más directos para llegar a la inteligencia del prójimo, y para acomodar el genio a los espíritus más ordinarios, sin necesidad de rebajarse. Fue de los antiguos de quien Melanchthon tomó sus planes, su pulcritud en la exposición, el arte de agrupar las pruebas, de proporcionar un tema, así como la claridad, esta luz que no ilumina a todo el mundo de la misma manera, pero que no deja a nadie en la oscuridad: la naturalidad en la expresión, que no es otra cosa que el lenguaje más general y apropiado (Nisard, 2010: 374$)^{96}$.

Este uso fácil y asequible de la comunicación, por parte de Melanchthon, facilitaba la transmisión de los saberes a la gente, complementando -por una parte- el modo comunicativo de Lutero que, aun siendo también eficaz, se expresaba dominantemente en términos teológicos y de forma intensa y rompedora, y -por otra parte- concretando mucho más que Erasmo su accesibilidad a todo el mundo, puesto que en este último su interés por ser un hombre universal le restó fuerza comunicativa. Es pertinente citar en este contexto la frase que Lutero escribió con tiza en la puerta de su casa, para expresar su percepción de lo que Melanchthon, Erasmo y él mismo significaron en el proceso de la reforma (letras y religión): «Res et verba Philippus, Verba sine re Erasmus. Res sine verbis

$\begin{array}{ll}{ }_{94} & \text { Op. cit. } \\ { }_{9} & \text { Op. cit. } \\ { }_{96} & \text { Op. cit. }\end{array}$

(C) EDICIONES UNIVERSIDAD DE SALAMANCA 
Lutherus» («La materia y las palabras, Philipp [Melanchthon]. Las palabras sin la materia, Erasmo. La materia sin las palabras, Lutero» [Stroh, 2012: 224-225] ${ }^{97}$.

Es más, en Melanchthon la fuerza de su comunicación radicaba en la fidelidad a la tradición clásica, constituyéndose en la referencia central de su vida, optando por la sinceridad y las cosas esenciales en sus actitudes, comportamiento y obra hacia la Reforma. En cambio, tanto Erasmo como Lutero -aun participando ambos del espíritu humanista-, se vieron mediatizados en su comunicación, bien por la tradición cristiana en el caso de Erasmo, o por la razón teológica, en el caso de Lutero; esto conllevó, en los dos casos, una pérdida de peso de las letras antiguas como soporte del proceso reformador.

El hecho de que para Melanchthon las Letras y los grandes escritores de la Antigüedad fueran su forma de vida, y que su preocupación principal se centrara en saber transmitir y adaptar los saberes a la gente y, más concretamente, a la juventud, explican el reconocimiento público que Alemania le hizo, nombrándole Praeceptor Germaniae, ya que «[...] hacía por la filosofía propiamente dicha, por la enseñanza de las lenguas, por la jurisprudencia, por la medicina, por las ciencias físicas, lo mismo que Lutero había hecho por la teología» (Nisard, 20Io: 379) ${ }^{98}$.

No obstante, puede decirse que posiblemente el profesor Melanchthon humanista, por muy erudito y brillante que fuera, tanto en Tubinga como en Wittenberg, hubiera pasado a la historia por ser conocido no más allá de dichos espacios universitarios o, en todo caso, no más allá de Alemania. En cambio, la figura de Melanchthon se proyectó mucho más allá, a toda la Europa humanista y de la Reforma, gracias a su vinculación a Martín Lutero y al papel que jugó en el desarrollo y en la organización de la Iglesia reformada.

97 Op.cit.

98 Op. cit. 